\title{
Chapter 2 \\ TEM Study of the Radular Teeth of the Chiton Acanthopleura japonica
}

\author{
Mitsuo Kakei, Masayoshi Yoshikawa, and Hiroyuki Mishima
}

\begin{abstract}
The radula chiton teeth, Acanthopleura japonica, were examined using transmission electron microscopy (TEM). After cutting into segments corresponding roughly to three developmental stages from the onset of tooth development, the middle and the fully matured stages, toluidine blue staining has given the posterior side three different color patterns, colorless, reddish-brown, and black colors, respectively. At the colorless stage, the microvilli attached along the surface of the tooth cusp appeared to be dissembled and convert into the lamellar structure in the tooth interior. At the reddish-brown stage, the electron density between fibrous layers increased. A complex of tiny clusters of grains appeared along the fibrous layers. They seemed to aggregate each other to become larger. At the black stage, multiple layers consisting of irregular-shaped and various size of iron minerals were formed. After treating with an aqua regia solution, organic substances have remained between iron minerals, suggesting the abrasion-resistant role at the posterior side of chiton teeth during feeding. In addition, these minerals were randomly arranged. The lattice intervals of the ion minerals varied at an approximate range from 4.8 to $10.2 \AA$. Also, we have confirmed clearly the lattice fringe of apatite crystal in the core region.
\end{abstract}

Keywords Chiton · Transmission electron microscopy · Iron minerals · Apatite crystal

The original version of this chapter was revised. A correction to this chapter is available at https://doi.org/10.1007/978-981-13-1002-7_44

M. Kakei ( $\square)$

Tokyo Nishinomori Dental Hygienist College, Tokyo, Japan

e-mail: mkakei@jcom.home.ne.jp

M. Yoshikawa

Division of Orthodontics, Meikai University School of Dentistry, Sakado, Japan

e-mail: ym-ortho@dent.meikai.ac.jp

H. Mishima

Department of Dental Engineering, Tsurumi University School of Dental Medicine,

Yokohama, Japan

e-mail: mishima-h@tsurumi-u.ac.jp 


\subsection{Introduction}

The lateral teeth of the radula exhibit a sequential series of tooth developments along with the production of iron biominerals from the organic stage to the fully mineralized stage. Regarding the iron biominerals in the radula teeth of the chiton, the teeth were composed of multilayers of iron oxide, predominately in the form of magnetite, but also in other forms of ferrihydrite and lepidocrocite (Lowenstam 1967). Therefore, many studies have focused mainly on these iron biominerals in the tooth cusp (Lowenstam 1967; Kim et al. 1986; Weaver et al. 2010; van der Wal 1989; Martin et al. 2009; Han et al. 2011). The tooth cusps of posterior side are mainly reinforced by the mineralization of magnetite at the matured stage. Due to its hardness, TEM study conducted by making thin sections has not been available in particular for the fully matured chiton teeth so far. On the other hand, the presence of a calcium phosphate mineral in the tooth core region has been examined using various techniques for a considerable time (Lowenstam and Weiner 1985; Kim et al. 1986; Evans et al. 1992; Evans and Alvarez 1999; Lee et al. 2000). Although a study using electron microscopy has been tried to demonstrate the presence of apatite crystal (Evans et al. 1992), the precise structure of apatite crystal has not yet fully elucidated. In this study, we have conducted the present study to demonstrate the unique iron mineral deposits and verify the detailed structure of apatite crystal mineral using an electron microscope.

\subsection{Materials and Methods}

Samples of the chiton Acanthopleura japonica were collected at Hachijojima's coastal area, Tokyo, Japan. Radulae were extracted from the chiton. After the removal of the soft tissue, radulae were cut into segments corresponding roughly to the three developmental stages of radula teeth from the onset of tooth development, the middle, and the fully matured stages, by using a razor blade. Then samples were subjected to examine using transmission electron microscope. They were fixed with $2 \%$ glutaraldehyde in $0.1 \mathrm{M}$ cacodylate buffer at $\mathrm{pH} 7.4$ for $1 \mathrm{~h}$ at $5{ }^{\circ} \mathrm{C}$, post-fixed with $1 \%$ osmium tetroxide in the same buffer for $1 \mathrm{~h}$ at $5{ }^{\circ} \mathrm{C}$, dehydrated by passage through a series of ascending ethanol concentrations, and then embedded in Araldite 502. Thick sections were stained with toluidine blue solution. Based on different color patterns at the posterior side of ralular teeth, these developmental stages tentatively called the initial stage with colorless, the middle stage with reddish-brown, and the fully matured stage with black color were examined. Thin sections were obtained with a Porter Blum MT2 ultra-microtome (SORVALL) equipped with a diamond knife. Sections were stained with saturated uranyl acetate and lead citrate, and some were left unstained. Also, some treated with an aqua regia solution were subjected to study. Then, they were examined under a JEM 100CX electron microscope (JEOL) at an accelerating voltage of $80 \mathrm{kV}$. 


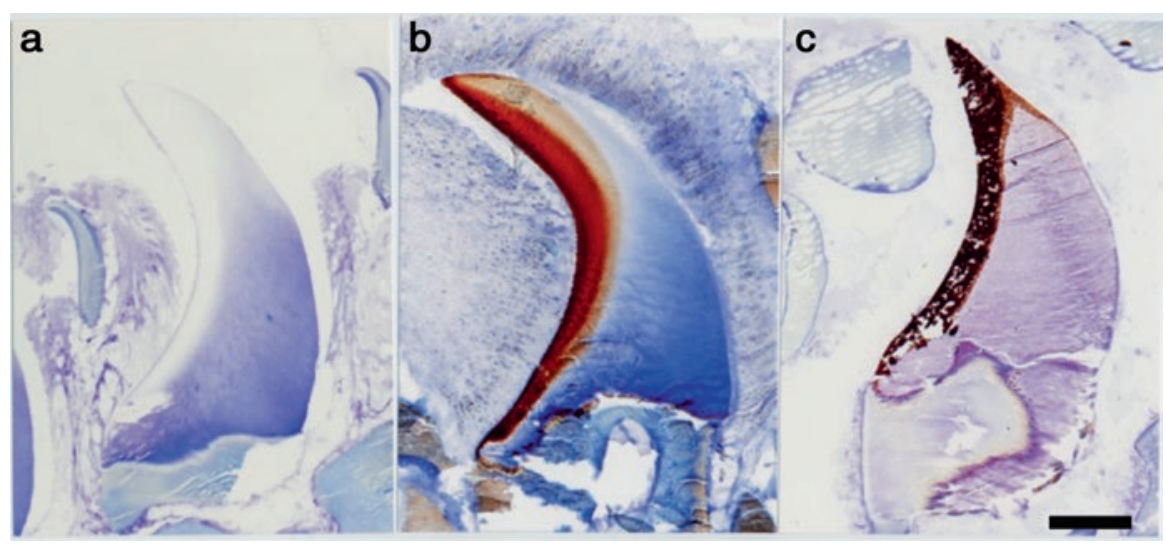

Fig. 2.1 Toluidine blue staining of the radula chiton teeth at three different stages. The change of color patterns at the posterior side reflects the degree of iron mineralization process. (a) The initial stage, (b) the middle stage, and (c) the fully matured stage. Toluidine blue-stained sections. Bar $=150 \mu \mathrm{m}$

\subsection{Results and Discussion}

Toluidine blue staining showed three different color patterns at the posterior side of three developmental stages from the initial stage with colorless, the middle stage with reddish-brown, and the fully matured stage with black color, respectively (Fig. 2.1).

At the colorless stage of the onset of tooth development, the internal structure of tooth cusps was filled with fibrous layers arranging in relatively parallel to the tooth surface at the anterior side (Fig. 2.2a). On the other hand, at the posterior side, fibrous layers bent upward adjacent to the inside of tooth cusp and run through toward the tooth tip (Fig. 2.2b).

At the reddish-brown stage of horizontal section, it was noted that higher magnifications of unstained sections have demonstrated that the deposits of ion minerals appeared immediately and grew more quickly at the posterior side than at the anterior side. So, it is suitable to examine the mineral deposits at the anterior side of the tooth cusp. A comparison between stained and unstained sections showed that the electron-dense zones were sandwiched by electron-lucent fibrous layers (Fig. 2.3). This suggests that the electron-dense zones might store mineral ions. At this stage, fibrous layers were altered to be the plume structure (Fig. 2.4a). Double-stained section showed the small and discrete particles were developed along fibrous layers. Also, it have been observed that a complex of tiny clusters of fine grains showing different electron density, which looked like a bunch of grapes, developed associating with the plume structure (Fig. 2.4b). The developed fine grains showing different electron densities seemed to aggregate each other and increase its size to create larger nonuniform grains. 

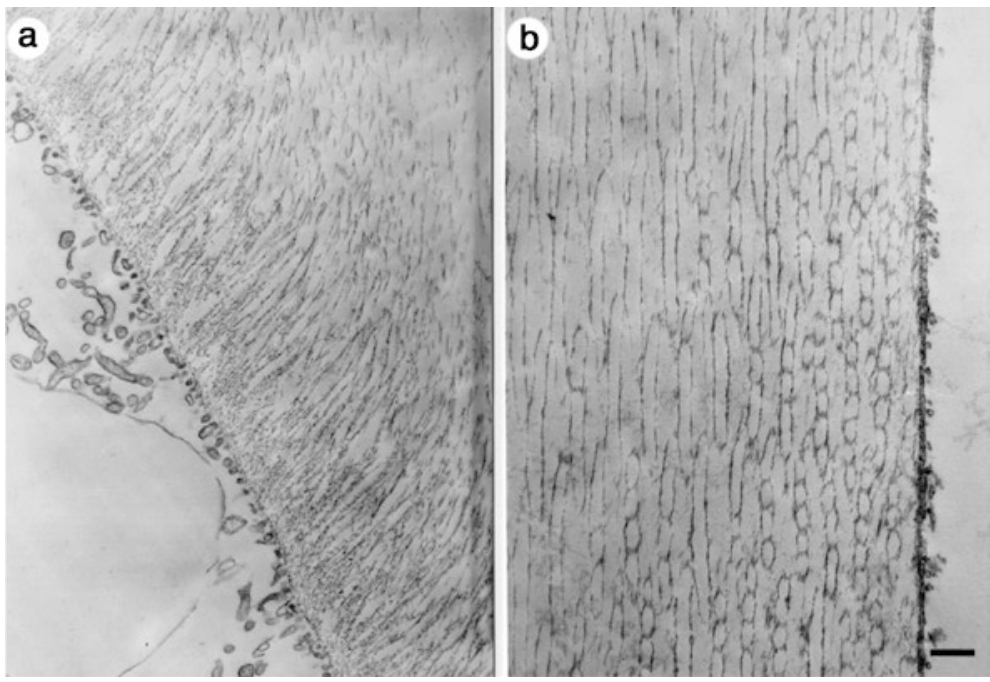

Fig. 2.2 TEM observations of the initial stage of chiton tooth. The arrangement of fibrous layer in the anterior side is different from posterior side of tooth cup. Double-stained sections. Bar $=0.5 \mu \mathrm{m}$
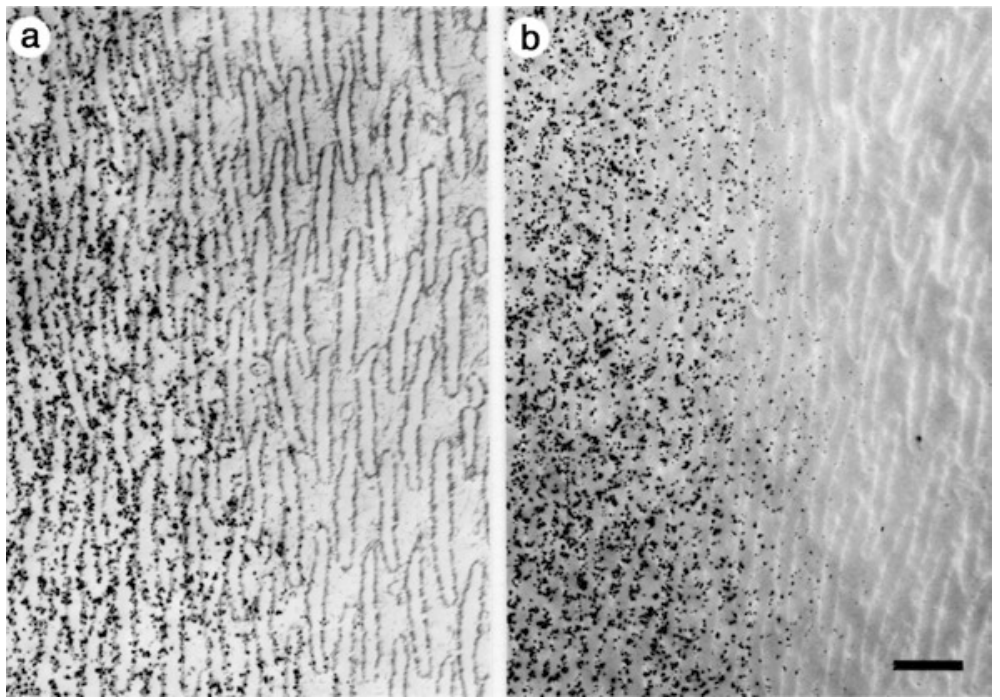

Fig. 2.3 TEM observations of the middle stage. By comparing stained with unstained sections, the space between organic layers shows a relatively high electron density. Double-stained (a) and unstained (b) sections. Bar $=1.0 \mu \mathrm{m}$ 


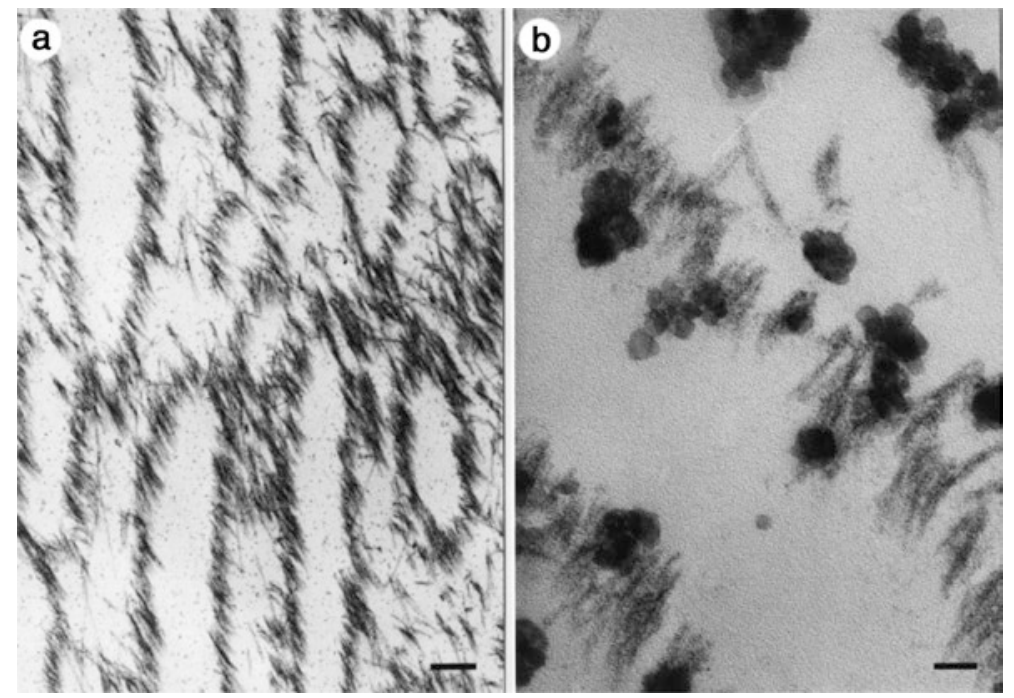

Fig. 2.4 High magnification of the plume structure (a) and the development of iron mineral grains (b) at the middle stage. The fine grains of iron minerals develop along the plume structure (b). Double stain. Bars $=100 \mathrm{~nm}(\mathbf{a}), 2.0 \mu \mathrm{m}(\mathbf{b})$

At the black color stage of longitudinal sections, TEM study has demonstrated that the multilayers of iron minerals at the posterior side showed a brick-like or veneer-like wall structure (Weaver et al. 2010) (Fig. 2.5a). Each iron layer seemed to be comprised of relatively large minerals (Fig. 2.5b). Also, it was noted that small minerals were scattering in distribution. After treating with an aqua regia solution, organic substances remained between iron minerals (Fig. 2.6a, b). Although the role of organic matrix is not fully elucidated, it has been considered that the organic matrix may control the mineralization processes (van der Wal et al. 2000; Nemoto et al. 2012) and contribute to resist crack and increase the tensile strength and flexibility during the feeding by the teeth (Evans et al. 1990; van der Wal et al. 2000).

Iron minerals observed near the core (Fig. 2.7a, b) and in the magnetite (Fig. 2.7c, d) regions are shown in Fig. 2.7. Regarding the lattice fringes of iron minerals, the estimate of lattice intervals of these iron minerals was ranging from 4.8 to $10.2 \AA$, approximately. On the basis of the observation at a high magnification of Fig. 2.5b, it has been considered that small iron minerals might gather together to create a lump of iron minerals. It is also considered that random arrangement of iron minerals could prevent the radula teeth from becoming magnetic and attracting ion sand which comes from the sandy beach.

TEM observation has clearly demonstrated the crystal fringe of apatite crystal in the core region (Fig. 2.8). Whether the crystals are fluorapatite have been discussed previously (Lowenstam 1967; Kim et al. 1986; Evans et al. 1992; Evans and Alvarez 1999; Lee et al. 2000). To our knowledge, the biologically induced apatite crystals are divided into central dark line (CDL)-free and CDL-bearing types (Kakei et al. 2016). Viewing from the CDL-free type of crystal structure in the core region of the chiton teeth, we assumed that fluorapatite was formed. 


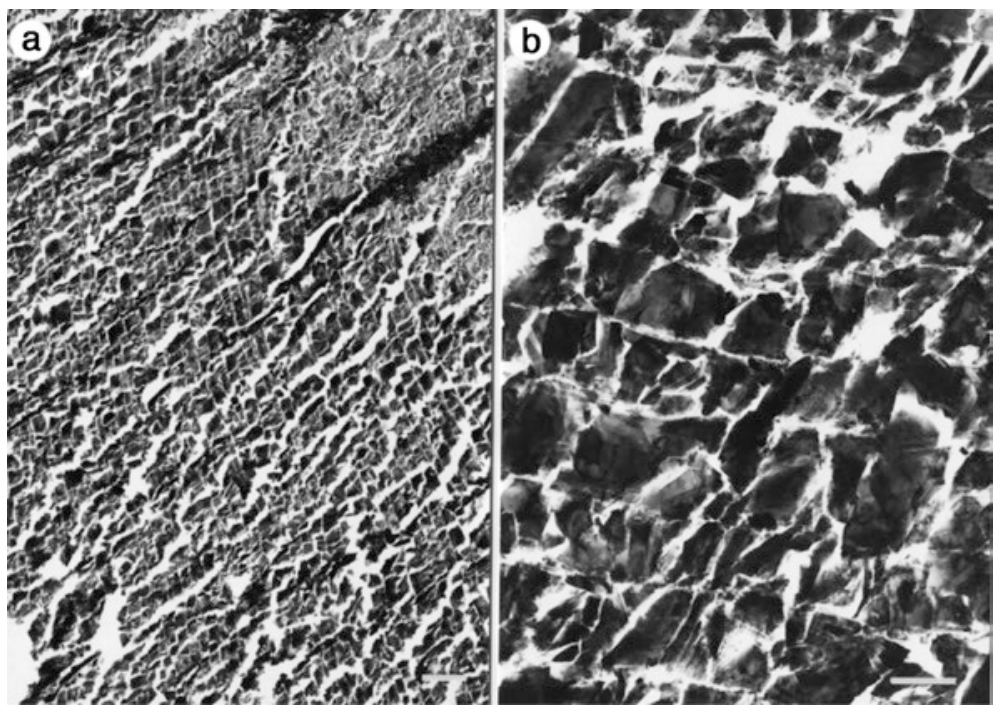

Fig. 2.5 TEM observations of the posterior region at the fully matured stage. The mineral layers consist of a brick-like wall or so-called veneer structure at the posterior side (a). Each mineral layer consists of both large angular and small minerals (b). No stain. Bars $=1.0 \mu \mathrm{m}(\mathbf{a}), 200 \mathrm{~nm}(\mathbf{b})$

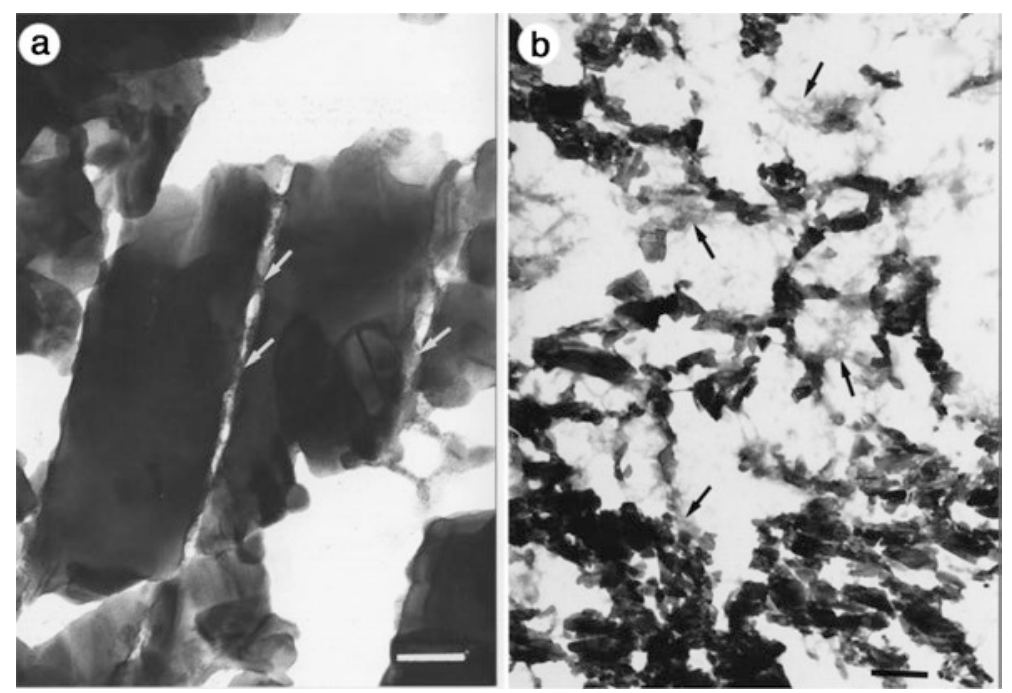

Fig. 2.6 TEM observations of aqua regia solution-treated sections. Treating with an aqua regia solution shows organic substances remained between iron minerals and suggesting a possible role of glue. (a) 5 min treatment, (b) 3 min treatment. Arrows indicate organic substances. Doublestained sections. Bar $=100 \mathrm{~nm}(\mathbf{a}), 20 \mathrm{~nm}(\mathbf{b})$ 


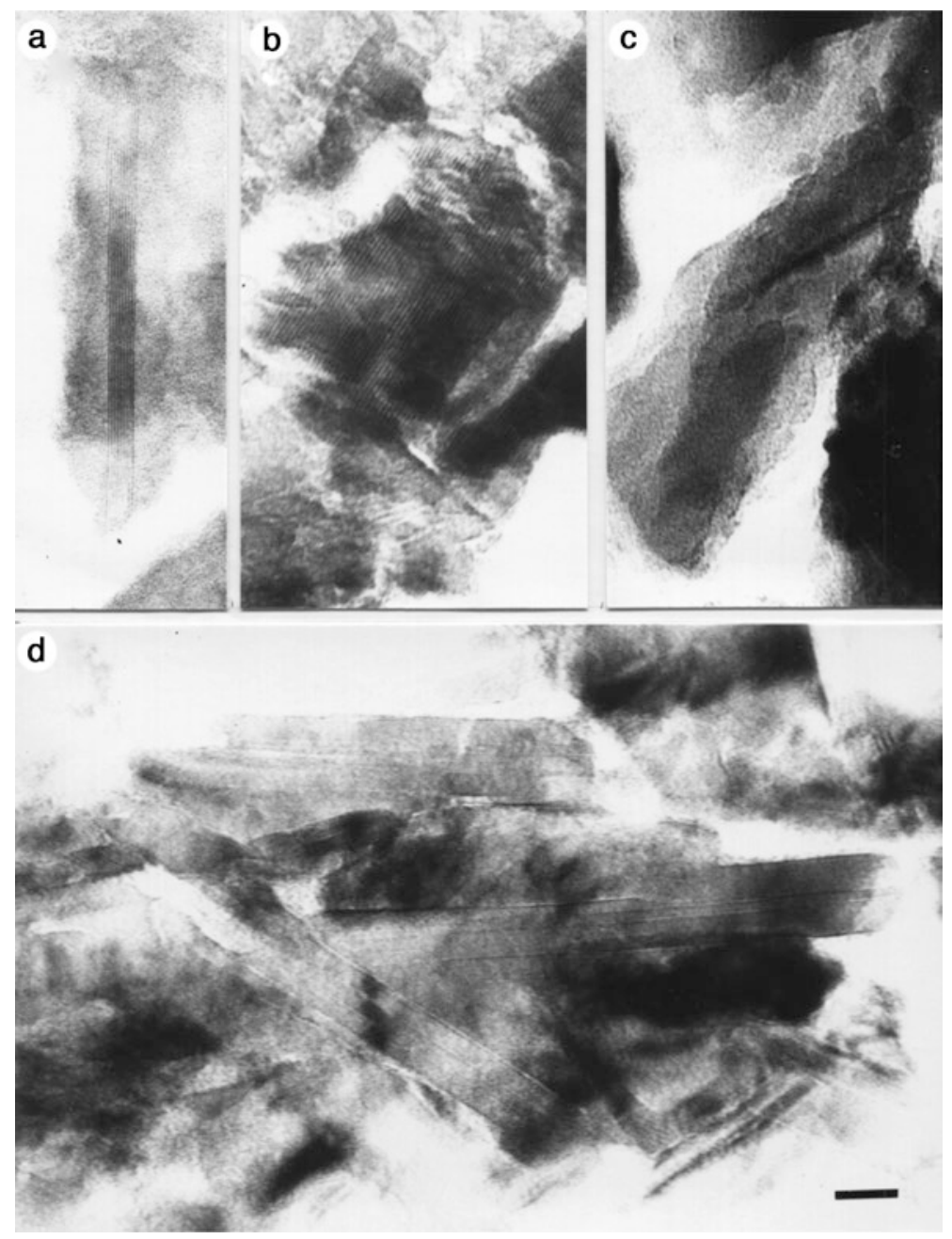

Fig. 2.7 TEM observations of iron minerals in the posterior region. Lattice fringes of iron minerals are recognized $(\mathbf{a}-\mathbf{d})$. Iron minerals are randomly arranged $(\mathbf{d})$. No stain. Bar $=10 \mathrm{~nm}$ 


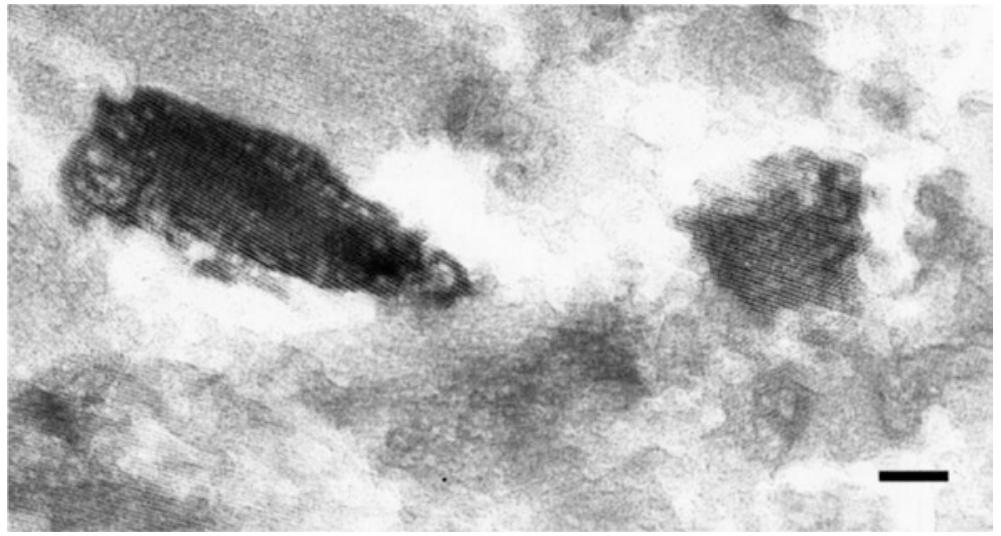

Fig. 2.8 TEM observations of calcium phosphate mineral in the core region. Two apatite crystals are confirmed. No stain. Bar $=10 \mathrm{~nm}$

\section{References}

Evans LA, Alvarez R (1999) Characterization of the calcium biomineral in the teeth of chiton Pellisepentis. JBIC 4:166-170

Evans LA, Macey DJ, Webb J (1990) Characterization and structural organization of the organic matrix of the radular teeth of the chiton Acanthopleura hirtosa. Philos Trans R Soc Lond B 329:87-96

Evans LA, Macey DJ, Webb J (1992) Calcium biomineralization in the radula teeth of the chiton, Acanthopleura hirtosa. Calcif Tissue Int 51:78-82

Han Y, Liu C, Zhou D, Li F, Wang Y, Han X (2011) Magnetic and structural properties of magnetite in radular teeth of chiton. Bioelectromagnetics 32:226-233

Kakei M, Yoshikawa M, Mishima H (2016) Aspects of the apatite crystal: two pathways for apatite formation, the mechanisms underlying crystal structure defects, and the pathological calcification event. J Fossil Res 48(2):53-65

Kim K-S, Webb J, Macey DJ, Cohen DD (1986) Compositional changes during biomineralization of the radula of the chiton Clavarizona hirtosa. J Inorg Biochem 28:337-345

Lee AP, Brooker DJ, Macey DJ, van Bronswijk W, Webb J (2000) Apatite menralization in teeth of the chiton Acanthopleura echinata. Apatite mineralization in teeth of the chiton Acanthopleura echinata. Calcif Tissue Int 67:408-415

Lowenstam HA (1967) Lepidocrocite, an apatite mineral, and megnetite in teeth of chiton (Polyplacophora). Science 156:1373-1375

Lowenstam HA, Weiner S (1985) Transformation of amorphous calcium phosphate to crystalline dahllite in radular teeth of chitons. Science 227:51-53

Martin S, Kong C, Shaw JA, Macey DJ, Clode P (2009) Characterization of biominerals in the teeth of the chiton, Acanthopleura hirtosa. J Struct Biol 167:55-61

Nemoto M, Wang Q, Li D, Pan S, Matsunaga T, Kisailus D (2012) Proteomic analysis from the mineralized radular teeth of the giant Pacific chiton, Cryptochiton stelleri (Mollusca). Proteomics 12:2890-2894 
van der Wal P (1989) Structural and material design of mature mineralized radula teeth of Patella vulgata (Gastropoda). J Ultrastruct Mol Struct Res 102:147-161

van der Wal P, Gleasen HJ, Videler JJ (2000) Radular teeth as models for the improvement of industrial cutting device. Mater Sci Eng C Biomim Supramol Syst 7:129-142

Weaver JC, Wang Q, Miserez A, Tantuccio A, Stromberg R, Bozhilov KN, Maxwell P, Nay R, Heier ST, DiMasi E, Kisailus D (2010) Analysis of an ultra hard magnetic biomineral in chiton radular teeth. Materialstoday 13 (1-2):42-52

Open Access This chapter is licensed under the terms of the Creative Commons Attribution 4.0 International License (http://creativecommons.org/licenses/by/4.0/), which permits use, sharing, adaptation, distribution and reproduction in any medium or format, as long as you give appropriate credit to the original author(s) and the source, provide a link to the Creative Commons license and indicate if changes were made.

The images or other third party material in this chapter are included in the chapter's Creative Commons license, unless indicated otherwise in a credit line to the material. If material is not included in the chapter's Creative Commons license and your intended use is not permitted by statutory regulation or exceeds the permitted use, you will need to obtain permission directly from the copyright holder.

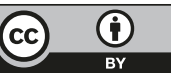

